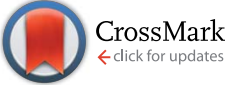

Cite this: RSC Adv., 2017, 7, 15964

\title{
A 2D approach to surface-tension-confined fluidics on parylene $\mathrm{C} \uparrow$
}

\author{
Paola Calcagnile, ${ }^{\star a}$ Tommaso Dattoma, ${ }^{a}$ Elisa Scarpa, ${ }^{a}$ Antonio Qualtieri, ${ }^{a}$ \\ Laura Blasi, ${ }^{\mathrm{b}}$ Massimo De Vittorio ${ }^{\mathrm{ac}}$ and Francesco Rizzi ${ }^{\star a}$
}

In the present study a new, facile and cheap method to obtain a 2D surface-tension-confined fluidic system on substrates conformally coated by parylene $C$ is presented. It is based on the use of poly(dimethylsiloxane) (PDMS)based soft masks obtained by molds produced by a 3D-printer. These masks, applied alternatively onto a parylene C-coated silicon substrate together with appropriate plasma treatments permit to obtain a superhydrophilic pattern on a superhydrophobic background, in which pure water, water-based solutions and polar solvents can flow. The flow of these liquids is strictly confined and is driven into the superhydrophilic pattern only by the differences in surface energy between it and the background, without any confinement effect provided by walls or capillary-driven channel, that are completely missing. According to the proposed fabrication method, all the desired fluidic systems can be fabricated in an easy and cheap way. The developed method for 2D surface-tension-confined fluidics on parylene $C$ permits to obtain a highly versatile platform which can be applied on all desired substrates, without the need to etch the polymer surface in order to obtain channel walls, paving the way to employ green, easily available and cheaper substrates, such as cellulose for paperbased fluidics applications, improving, at the same time, the biopolymer surface properties.

Received 8th February 2017

Accepted 7th March 2017

DOI: $10.1039 / \mathrm{c} 7 \mathrm{ra01604b}$

rsc.li/rsc-advances
Control of wettability of surfaces by tuning superhydrophilicity/superhydrophobicity properties in patterns could be employed as an alternative strategy for realizing fluidics. Superhydrophilic and superhydrophobic surfaces in nature have continuously inspired biomimetic designs to manipulate material surface wettability. ${ }^{3-8}$ The opportunity to fabricate both types of surfaces has been receiving profound attention due to a broad applicability and thus industrial importance. ${ }^{9-13}$

The challenge is the production of both superhydrophilic and superhydrophobic areas, characterized by extreme difference in surface energy, on the same sample. This task can be extremely advantageous in many applications, and it has been demonstrated for droplet or cell manipulation, ${ }^{\mathbf{1 2 , 1 4}}$ channels for peptide separation, ${ }^{15}$ or microcondensation. ${ }^{16}$ The emerging application in this context is the creation of $2 \mathrm{D}$ and $3 \mathrm{D}$ surfacetension-confined (STC) fluidics, where the difference in wettability properties, namely surface tension, confines and drives liquids. ${ }^{17-19}$ Specifically, STC fluidic devices confine liquids in surface portions characterized by higher surface energy compared to the adjacent ones, eliminating the use of pumps and valves and allowing the easy introduction of liquids into the device by simple and fast methods, such as, for instance, with a pipette, thus representing an eco-friendly and low-energy consumption method to transport fluids.

If controlling surface wettability is possible, an STC fluidics is very convenient to be applied in both traditional and lab-onpaper technologies without the need of any actuation system for microfluidics. A possible strategy to be pursued is based on 
parylene $\mathrm{C}$, being a conformal coating which can be easily extended to any substrate. Parylene $\mathrm{C}$ is a versatile polymer, characterized by dielectric insulating properties, chemical resistance and inertness, poor permeability to moisture and corrosive gases. Moreover, it can be deposited onto various substrates by Room Temperature-Chemical Vapour Deposition (RT-CVD), providing a transparent, flexible, inert and room temperature available conformal coating on any material of any shape. For these qualities, parylene $\mathrm{C}$ has emerged as potential material not only as insulator conformal coating for flexible electronics, ${ }^{20}$ but even for biomedical applications, sensors and microfluidics. ${ }^{21-25}$

Creating surfaces with patterned superhydrophilic/ superhydrophobic domains, accordingly to a specific topography, depends on surface chemistry, morphology and the capability to induce such properties onto the surface. ${ }^{26,27}$ At the same time, microscopic and submicroscopic structures, to enhance surface roughness, can be grown from solutions ${ }^{28}$ or through lithographic and electrochemical ${ }^{\mathbf{2 9}, 30}$ routes. The creation of micro/nanostructure is also achieved by deposition of films of nanoparticles by ink-jet printing, ${ }^{31}$ by sol-gel techniques, ${ }^{32}$ sputtering, ${ }^{33}$ spin coating ${ }^{34}$ or drop casting. ${ }^{35}$ Creating patterns of surface wetting properties has been continuously inspiring the research about fluidics and engineered surfaces. ${ }^{36-38}$

In this scenario, plasma-based modification methods show many advantages if compared to other techniques. First of all, they are able to modify the surface properties of the treated samples without altering the bulk characteristics; moreover, they are performed in a temperature-controlled and dry environment, thus permitting the treatment of heat-sensitive materials and avoiding the use of solvents or other toxic reagents. ${ }^{39,40}$ Plasmabased surface treatments are also very versatile, as they can be used for a broad class of crystalline and polymeric materials, providing different effects onto the treated surfaces. Indeed, the induced modifications can be chemical, through the introduction of new chemical groups or functionalities and/or the deposition of thin functional coatings, or physical, through the etching and texturing of the surface. ${ }^{\mathbf{4 1 , 4 2}}$ Additionally, plasma treatments are characterized by ease and speed of performance.

In this study, a facile and innovative method to obtain a superhydrophilic pattern in a superhydrophobic background on parylene $\mathrm{C}$ is presented. The proposed method is based on the use of complementary poly(dimethylsiloxane) (PDMS)-based soft masks, made by casting in 3D-printed molds and applied alternatively onto the parylene surface, in combination with specific plasma treatments. The masks, exhibiting appropriate geometry, are able to produce the desired pattern on parylene surface, thus creating a STC fluidic system, where water, waterbased solutions and polar solvents can flow, driven only by the difference in surface tension. Moreover, the explored route permits to obtain $2 \mathrm{D}$ patterns onto the polymer surface, and therefore the liquid drops are self-driven and confined along the surface of a bidimensional path without tridimensional sidewall. As the flow velocity of a solution is an important parameter to be tested in STC fluidic systems, in this work it was calculated for different water/ethanol mixtures through the channel.
To the best of our knowledge it is the first time that parylene $\mathrm{C}$ surface has been modified to obtain a STC fluidic system. Due to the ease of processing, such a parylene C-based STC fluidic device can be used for chemical reactions, spot test analysis, actuators, sensors, or in all those fluidic applications where the insulating and resistant coating provided by parylene $\mathrm{C}$ is necessary. Moreover, due to the conformal coating provided by parylene $\mathrm{C}$ and to the softness and flexibility of the PDMS masks, the optimized technique for STC fluidics on parylene can be reproduced on substrates of any shapes and materials not only silicon ones - thus permitting to use cheaper and easier to be processed materials. On this basis, the proposed technique can find a broad applicability in traditional and especially paper-based fluidics, where the insulating and moisture-resistant properties of parylene $\mathrm{C}$ are necessary to overcome the intrinsic limits of cellulose-based biopolymers, thus representing a friendly and no-energy consumption method to transport fluids in lab-on-paper.

\section{Experimental}

\section{Materials}

Silicon substrates $525 \mu \mathrm{m}$ thick, type 100, used as substrates for parylene $\mathrm{C}$ deposition, were supplied by Okmetic.

Parylene C (poly(para-xylylene)) was purchased as dimer powder from Specialty Coating Systems.

Poly(dimethylsiloxane) (PDMS) (Sylgard 184 Silicone Elastomer), used as mask in order to create distinct superhydrophilic and superhydrophobic patterns onto the parylene $\mathrm{C}$ surface, was supplied in two compounds, a pre-polymer and a crosslinker, by Dow Corning Corporation.

Experiments performed to check the flow velocity of different fluids onto the created STC channel were carried out using controlled mixtures of pure water treated through a Milli-Q Plus purification system and ethanol supplied by Sigma Aldrich.

\section{Parylene C deposition}

Parylene $\mathrm{C}$ belongs to the family of poly(para-xylylene) polymers and it is characterized by the presence of a chlorine atom in the aromatic monomers. Parylene $\mathrm{C}$ was deposited onto silicon substrates providing a conformal coating after a RT-CVD process achieved by Specialty Coating Systems, PDS 2010 Labcoater system model.

The detailed deposition process was performed as reported in previous literature..$^{24,25}$

\section{Fabrication of PlasPINK® molds and PDMS masks}

In order to produce two PDMS masks, one the negative of the other, two molds were primarily fabricated by means of the $3 \mathrm{D}$ printer ASIGA FREEFORM Pico Plus 27. The molds were realized by PlasPINK® UV-curable polymer and printed, layer by layer, by a MEMS projector based on a $405 \mathrm{~nm}$ UV LED.

The two molds were the complement of each other. Mold 1 exhibits the shape of a channel between two circular reservoirs deepened into a rectangular surrounding substrate (channel length $20 \mathrm{~mm}$, width $2 \mathrm{~mm}$, reservoir diameters $4 \mathrm{~mm}$, 
rectangular external area $35 \mathrm{~mm} \times 22 \mathrm{~mm}$ ). On the contrary, mold 2 exhibits the previous shape of the channel and the two circular reservoirs (with the same dimensions of mold 1) in relief with respect to the same surround on a continuous substrate. Starting from molds 1 and 2 it was possible to fabricate respectively mask 1 and mask 2 . Indeed, the degassed mixture of PDMS pre-polymer and crosslinker $(10: 1(\mathrm{w} / \mathrm{w})$ ratio) were poured into the two opposite molds and then cured at $110{ }^{\circ} \mathrm{C}$ for 5 minutes. Then, the polymerized PDMS was gently removed from the two molds, and consequently, the two negative masks were obtained: mask 1 exhibits the shape of a fluidic channel between two circular reservoirs; conversely, mask 2 exhibits the shape of the surrounding rectangular area deprived of the same channel.

The two masks produced in this way were used during the subsequent plasma processes on parylene $\mathrm{C}$ surface, to create a superhydrophilic channel in a superhydrophobic domain.

\section{Plasma surface treatments}

Different plasma treatments were optimized to pattern parylene C surface, rendering it selectively superhydrophilic or superhydrophobic, according to the employed PDMS masks. The processes were carried out in the Oxford Estrelas Plasma Pro 100 Inductively Coupled Plasma (ICP) etcher.

In order to make parylene $\mathrm{C}$ superhydrophilic, a single-step treatment was performed. As predicted by the Wenzel's equation, hydrophilicity is enhanced by plasma induced nanotexturing. This leads to superhydrophilicity for processing power and times showing the highest surface-to-volume ratio, increasing exposed surface and consequently the density of plasma-induced hydrophilic groups thereon. ${ }^{25}$ In the present work, we reproduced this higher surface-to-volume ratio with the following oxygen plasma condition: $1000 \mathrm{~W}$ and $150 \mathrm{~W}$ as powers of the coil and the platen, respectively, and a flow rate of $100 \mathrm{sccm}$, under a pressure of $20 \mathrm{mtorr}$ and a temperature of $10{ }^{\circ} \mathrm{C}$, for $30 \mathrm{~s}$. To render the polymer surface superhydrophobic, the following two-steps process was implemented: the first step consists of a preliminary oxygen plasma performed with $0 \mathrm{~W}$ and $150 \mathrm{~W}$ as powers of the coil and the platen, respectively, in order to roughen the parylene surface but limiting the plasma-induced hydrophilic groups thereon and a flow rate of $100 \mathrm{sccm}$, under a pressure of $20 \mathrm{mtorr}$ and a temperature of $10^{\circ} \mathrm{C}$, for $3 \mathrm{~min}$; the following second step consists of a $\mathrm{C}_{4} \mathrm{~F}_{8}$ deposition, achieved with $0 \mathrm{~W}$ and $900 \mathrm{~W}$ as powers of the platen and coil, respectively, a flow rate of $25 \mathrm{sccm}$, under a pressure of $60 \mathrm{mtorr}$ and a temperature of $0{ }^{\circ} \mathrm{C}$, for $1 \mathrm{~min}$.

\section{Fabrication of a 2D surface-tension-confined channel on parylene $\mathrm{C}$}

In order to create a $2 \mathrm{D}$-channel where water could flow driven exclusively by the differences of surface tension, the two described above plasma treatments were performed in sequence. In particular, the two-steps process was primarily applied onto the parylene $\mathrm{C}$ surface, using mask 1 , to render the surrounding area of the channel, exposed to plasmas, superhydrophobic. Before peeling off mask 1 from the sample, mask 2 was carefully aligned onto the portion of the surface already made superhydrophobic. Subsequently, mask 1 was removed and the exposed area was subjected to the single-step oxygen plasma process. In this way, the area corresponding to the channel was made superhydrophilic in a superhydrophobic environment and, therefore, a STC channel onto the surface of parylene $\mathrm{C}$ was obtained. A schematic representation of the entire process is available in Fig. 1.

\section{Sample characterization}

The thickness of the parylene $\mathrm{C}$ conformally deposited onto the surface of the silicon wafers was measured by means of a profilometer (Bruker Dektak Xt).

After the plasma surface treatments, the water contact angle (WCA) and the WCA hysteresis of parylene C surfaces were measured with a contact angle goniometer (KSVCAM200, Kruss, Germany), with droplets of distilled water of $2 \mu \mathrm{L}$.

For the measurements of water contact angle hysteresis, advancing and receding CAs were generated keeping fixed the drop volume $(2 \mu \mathrm{L})$ and gradually increasing the tilt angle applied to the samples, starting from flat position, until the instant before the drop slips away from the sample surface. The WCA hysteresis was recorded as the difference between right and left WCA.

All the reported values of WCAs and WCA hysteresis, with corresponding standard deviations, are the average of 5 independent measurements.

Morphological analysis of the plasma treated parylene surface was performed by means of a Scanning Electron Microscopy-Focused Ion Dual Beam (SEM-FIB) by FEI Helios NanoLab 600i DualBeam.

The flow velocity of water/ethanol mixtures in different percentage of water $(0,20,50,80,100 \%)$ on the fabricated STC channel was tested in order to check the behavior of liquids with different surface tension. It was calculated taking into account the length of the linear part of the fluidic system and the time in which the liquids go through it.

\section{Results and discussion}

In this paper, a facile approach for the fabrication of superhydrophilic patterns in a superhydrophobic background on parylene $\mathrm{C}$ is presented. It is based on the use of complementary PDMS-based masks fabricated via 3D-printing applied alternatively onto the parylene surface, in combination with specific plasma treatments.

3D fabrication technologies allow to quickly replicate tools and structures involving the exploitation of different materials ranging among resins, polymers and biological tissues. In particular, 3D-printers are a cheap and fast way to fabricate molds, providing also the opportunity to realize masks made in soft polymeric materials, such as PDMS. PDMS is a very suitable polymer to be used as a mask on parylene $\mathrm{C}$ substrates because, due to parylene anti-sticking properties, it lies on these substrates without altering the surface properties. ${ }^{22}$ For the same reason, PDMS was previously and successfully exploited in microcontact printing of parylene $\mathrm{C}$ coated surfaces. ${ }^{25}$ 
(1)

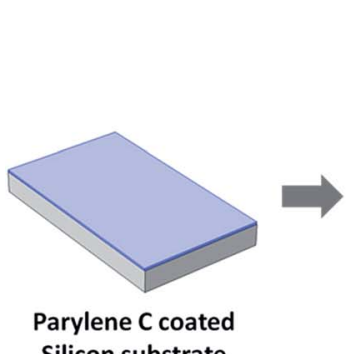

Two-steps process for

(2) Superhydrophobicity

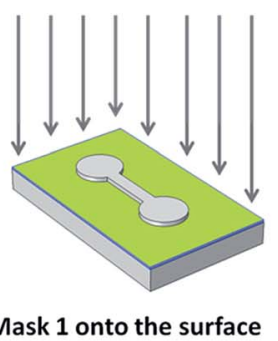

Mask 1 onto the surface

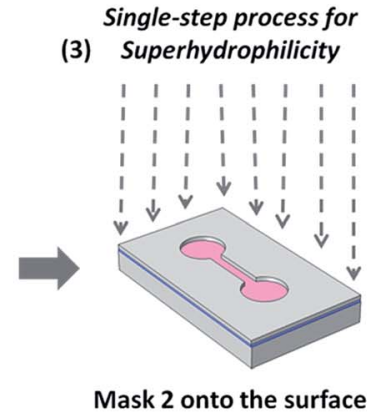

Fig. 1 Schematic representation of the developed plasma-based approach to induce a bidimensional surface-tension-confined channel onto a parylene $C$ surface. Parylene $C$ surface is represented in green, while the PDMS masks in blue. (1) Deposition of parylene C onto a silicon substrate via a RT-CVD process generating a conformal coating; (2) application of mask 1, exhibiting the shape of a channel, onto the parylene C surface and execution of the subsequent oxygen and $\mathrm{C}_{4} \mathrm{~F}_{8}$ plasma treatments for superhydrophobization; (3) application of mask 2 (negative with respect to mask 1) and execution of oxygen plasma process for hydrophilization; (4) resulting 2D-STC channel onto the polymer surface.

The masks, exhibiting appropriate geometry, are able to produce the shape of a fluidic channel on parylene surface, thus creating a so-called surface tension confined fluidic system, where water and water-based solutions can flow driven only by the difference in surface tension between the inner and the external parts of the channel. The flow is entirely confined and, beside surface tension, no other physical barrier works, because the obtained channel is a bidimensional system, devoided of any depth.

The substrates employed in this work are parylene C-coated silicon wafers. Untreated parylene $\mathrm{C}$ is a hydrophobic polymer, exhibiting a WCA of $88.8^{\circ} \pm 0.7^{\circ} .^{10}$ The first step for the fabrication of the presented fluidic system is the switching of the wettability properties of this polymer in two opposite directions, in order to make it alternatively superhydrophobic and superhydrophilic. The optimized single-step oxygen plasma treatment performed by ICP etcher leads to an effective superhydrophilic character of the surface, as the recorded WCA is $4.71^{\circ} \pm 0.21^{\circ}$. At the same time, the two-steps treatment based on subsequent oxygen and $\mathrm{C}_{4} \mathrm{~F}_{8}$ plasmas makes parylene completely superhydrophobic, with a WCA of $149.18^{\circ} \pm 1.20^{\circ}$. Wettability results are shown in Fig. 2a. The surface is rendered superhydrophobic but not self-cleaning, as the measured WCA hysteresis is $10.84^{\circ} \pm 0.5^{\circ}$, with advancing and receding CAs, at a tilt angle of $90^{\circ}, 129.87^{\circ} \pm 1.27^{\circ}$ and $119.03^{\circ} \pm 1.67^{\circ}$ respectively. This result matches the findings of a study by Teisala et $a .^{43}$ about nanostructured superhydrophobic surfaces, demonstrating that water penetrates into the nanofeatures, adhering to the surface. This special behavior is addressed as the "rose petal effect", ${ }^{44}$ differing from the "lotus leaf effect", because even if WCA shows a superhydrophobic behavior (WCA $\geq 150^{\circ}$ ), the water hysteresis is higher than $10^{\circ}$, and water drops remain attached onto the surfaces, with superhydrophobic and sticky properties at the same time.

Morphological analysis, performed by SEM, was carried out to verify the topographical effects due to the plasma treatments and the results are shown in Fig. 2. Untreated parylene $\mathrm{C}$ (Fig. 2b) surface is completely flat and the performance of the $\mathrm{C}_{4} \mathrm{~F}_{8}$ deposition process does not alter such a morphology
(Fig. 2c). On the contrary, oxygen plasma treatment is responsible of the induced roughness onto the polymer surface due to the etching phenomena taking place during the process (Fig. 2d), while, the subsequent application of $\mathrm{C}_{4} \mathrm{~F}_{8}$ plasma does not modify further the surface topography, as expected (Fig. 2e).

In Fig. 3 the plasma oxygen treated sample is shown at increasing magnification, clearly exhibiting the induced nanosized features on the previously flat parylene surface.

Superhydrophilicity of parylene C samples treated with the single-step oxygen plasma is due to a double effect, chemical and physical, induced by the ICP plasma treatment. Specifically, plasma provoked hydrophilicity is caused by the bonding of oxygen-based chemical groups onto the polymer surface, such as hydroxyls, aldehydes, carbonyls or carboxyls, which contributes to increase the surface energy. ${ }^{45-47}$ Seong et al. and Hoshino et $a .^{47,48}$ confirmed this feature with their X-ray photoelectron spectroscopy analysis performed on parylene $\mathrm{C}$ samples, revealing that a larger amount of oxygen and a smaller one of carbon atoms follows oxygen plasma treatments. Moreover, during the plasma process also the nanotexturing of the surface is induced $^{\mathbf{1 4}}$ via physical interaction with reactive ions, achieving surface superhydrophilicity according to Wenzel's wettability model. Finally, nanotexturing, increasing the exposed surface of the polymer, provides bigger area liable to chemical attack by hydrophilic oxygen-based groups. ${ }^{10,42,45-47,49-52}$

Also the opposite superhydrophobicity behavior is achieved by combining chemical and physical effects of plasmas. Indeed, the optimized two-steps process takes advantage of the nanoroughness induced by the preliminar oxygen plasma treatment to texture the surface and then, by the following fluorine-based plasma, to dramatically reduce parylene surface energy. It is well-known that flourine-based surfaces are hydrophobic and that can become superhydrophobic if some roughness is introduced in their morphology. ${ }^{\mathbf{1 0 5 3 - 5 6}}$ These findings are confirmed by the wettability characterization, as the WCA of the sample treated only with $\mathrm{C}_{4} \mathrm{~F}_{8}$ plasma is $119.23^{\circ} \pm 1.82^{\circ}$, thus merely hydrophobic. Consequently, it is the combination of 


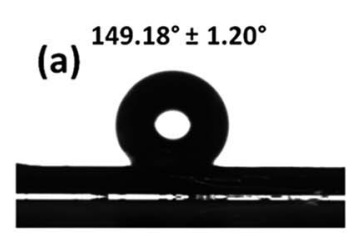

$4.71^{\circ} \pm 0.21^{\circ}$
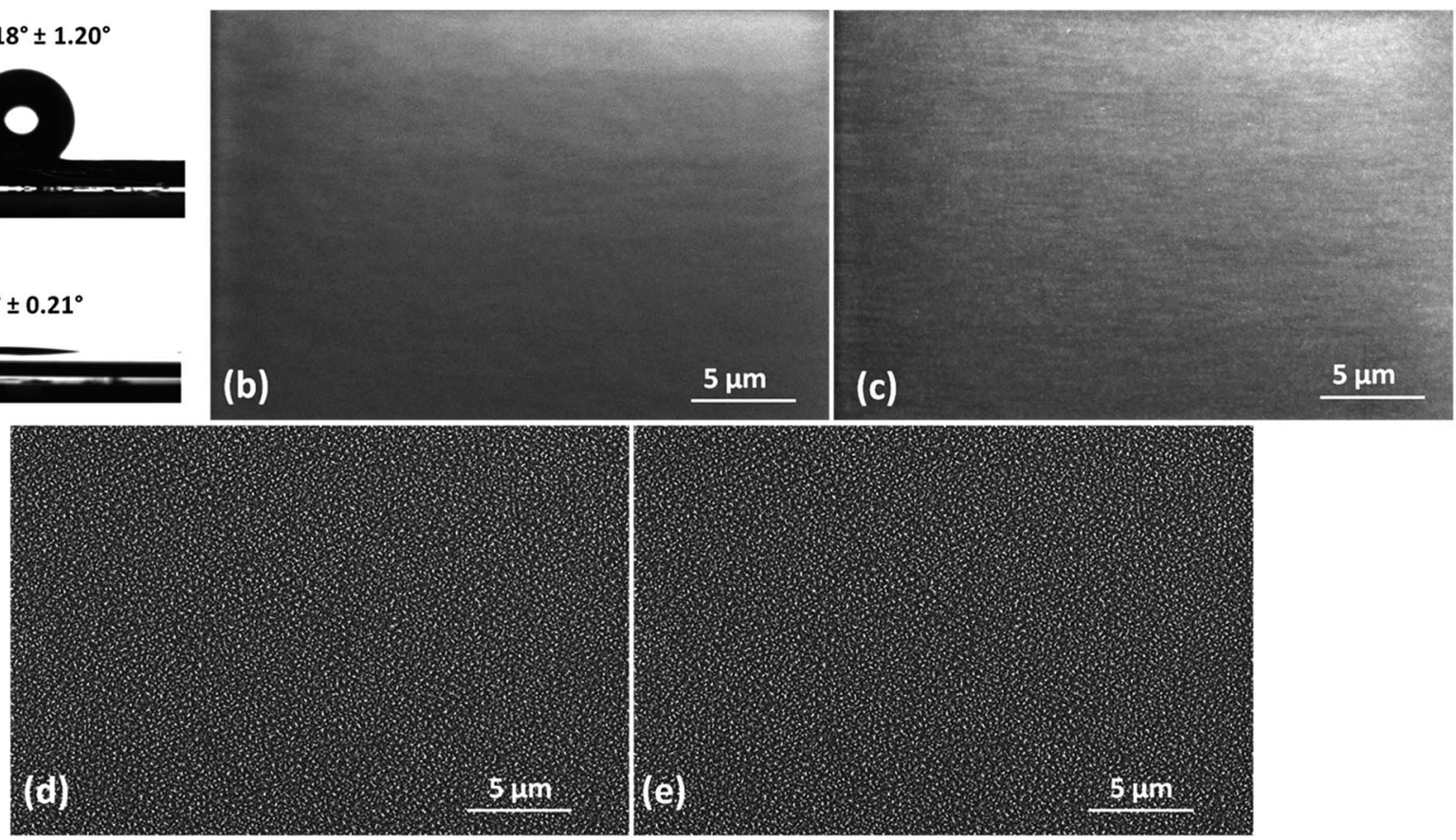

Fig. 2 Wettability and morphological data of the plasma treated samples. (a) Photos of WCAs of parylene $C$ surface rendered superhydrophilic via the single-step oxygen plasma treatment (on bottom) and superhydrophobic via the two-steps oxygen and $\mathrm{C}_{4} \mathrm{~F}_{8}$ plasma treatment (on top). (b) Pristine parylene $\mathrm{C}$ surface, (c) parylene $\mathrm{C}$ surface after $\mathrm{C}_{4} \mathrm{~F}_{8}$ plasma deposition, (d) parylene $\mathrm{C}$ surface after oxygen plasma etching, (e) parylene $\mathrm{C}$ surface after oxygen plasma and $\mathrm{C}_{4} \mathrm{~F}_{8}$ treatments.

chemical and texturing effects, provided by the two-steps process, which leads to superhydrophobic parylene C.

The fluidic system obtained according to the procedure described in Fig. 1 and its functioning are shown in Fig. 4, where the superhydrophilic/superhydrophobic character of the inner/external parts of the channel are clearly evident. Water is driven along the channel exclusively by the difference in surface energy between the inner and the external areas of the channel, without the confinement effect provided by any channel wall, completely absent. Moreover, water remains confined into the channel also after rotating the substrate (see the ESI $\dagger$ file).

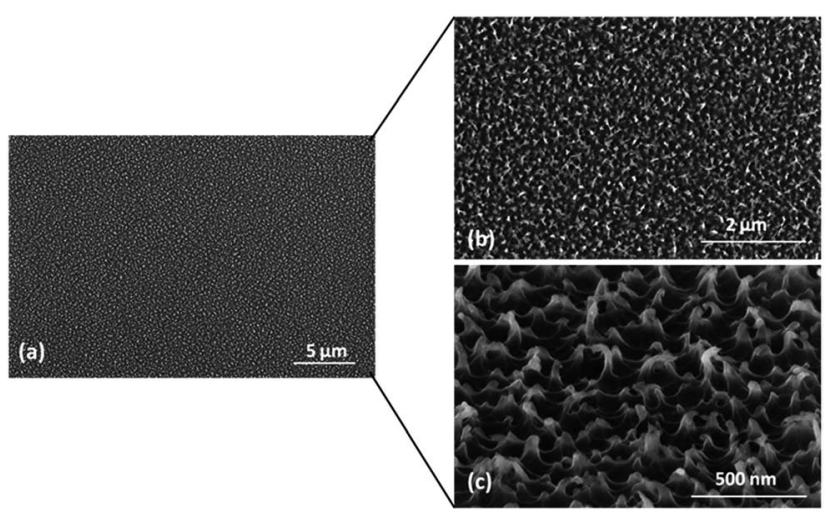

Fig. 3 SEM images at different magnification of the oxygen plasma treated sample. The nanosized induced texture is clearly visible onto the polymer surface.
In fluidic systems the flow velocity is an important parameter to be tested. The fabricated system was tested with a water/ ethanol mixtures composed by different volume percentage of water $(0,20,50,80,100 \%)$. The results of this analysis, shown in Fig. $5 a$, indicate that the flow velocity decreases while increasing water content, for lower than $50 \%$ amount of water, and it increases by increasing the amount of water in the mixtures, for

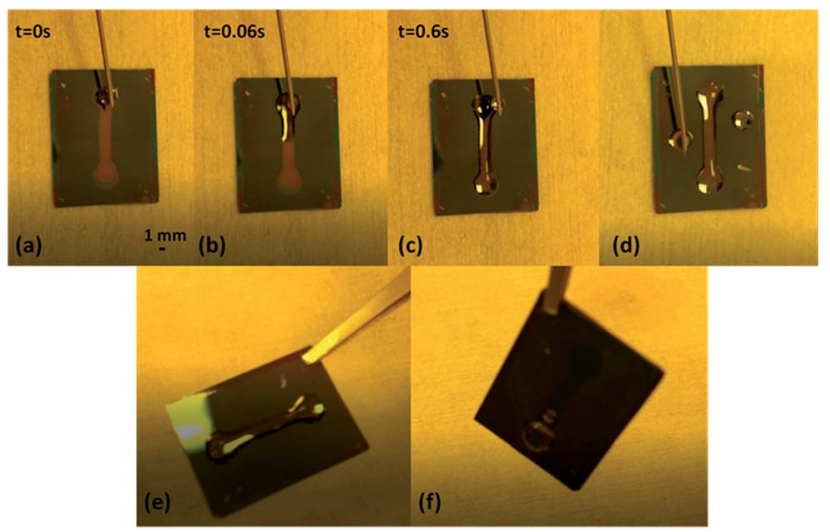

Fig. 4 Photos of the fabricated STC channel on parylene $C$ surface. $(a-c)$ Water flow into the channel at different time intervals $(t=0 \mathrm{~s}$, $0.06 \mathrm{~s}$ and $0.6 \mathrm{~s}$ ). A water drop is gently lied onto the first reservoir, spreading towards the second one, confined in the channel and driven only by differences in surface energy onto the substrate. (d) Superhydrophilic and superhydrophobic character of the surface inside and outside the channel. (e and f) While rotating the substrate of about $90^{\circ}$ water stays confined into the channel. The related videos are available in the $\mathrm{ESI} \uparrow$ file. 

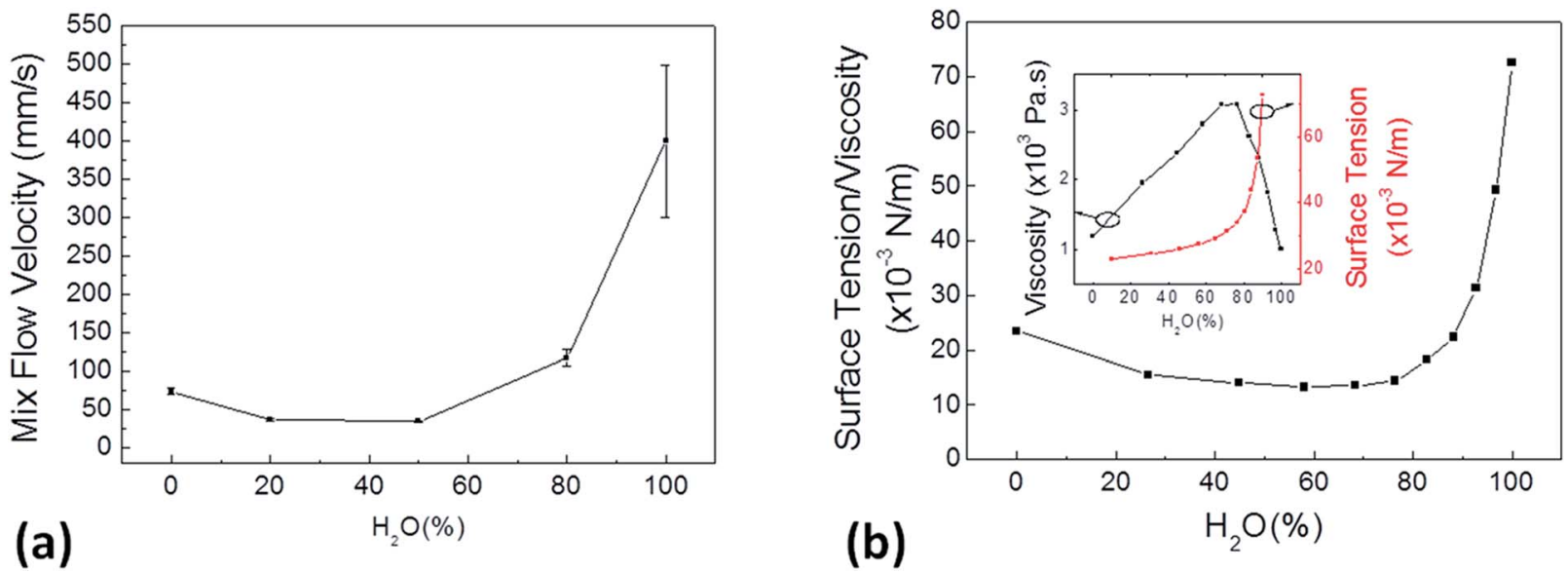

Fig. 5 Flow velocity measurements for the prepared water/ethanol mixtures and related physical/chemical properties. In (a) flow velocity measurements of the tested water/ethanol mixtures characterized by different percentage of water in the final volume. Velocity data points are characterized by increasing error bar as higher values of velocity are more susceptible to measurement error. (b) The surface tension/viscosity ratio of the same mixtures and at the same temperature $\left(20^{\circ} \mathrm{C}\right)$ according to the values reported in ref. 57 ; inset: surface tension and viscosity of water/ethanol mixtures at $20^{\circ} .{ }^{57}$ The trends shown in (a) and (b) are comparable.

higher water amount. These results can be interpreted if taking into account some physical parameters of the mixtures, specifically surface tension and viscosity. In case of a highly wetting mixture, as the water/ethanol mixtures on the obtained superhydrophilic surface, the velocity of a fluid into a channel is proportional to the surface tension/viscosity ratio at a fixed temperature (Schutzius et al. ${ }^{57}$ ), $20{ }^{\circ} \mathrm{C}$ in the present study. Indeed, the trend of the measured flow velocities clearly follows the surface tension/viscosity ratio of the same mixtures at the same temperature (Fig. 5b), in agreement with the measurements reported in Khattab et al. ${ }^{58}$ Surface tension of water/ ethanol mixtures increases with the water percentage, while viscosity exhibits a bell-shaped curve with a maximum at around $70 \%$ of the water content (inset in Fig. $5 \mathrm{~b}$ ). ${ }^{57}$ Consequently, in the range between $0 \%$ and $50 \%$, when the surface tension of the mixture is low, the viscosity effects have a great relevance in the surface tension/viscosity ratio: as viscosity increases, the ratio value decreases (as observed in the flow velocity measurements). In the range between $50 \%$ and $100 \%$ of water percentage range, the role of surface tension of water/ethanol mixture becomes more relevant than its viscosity and the surface tension/viscosity ratio follows a quick monotonous increasing trend; consequently, the flow velocity grows with the water percentage.

The velocities were also tested 12 hours after the first test, executed soon after the preparation of the samples. They reproduce the values recorded in the first test, with increased standard deviations (results not shown), probably due to the ageing effect of the plasma oxygen treatment. ${ }^{25}$

\section{Conclusions}

An easy and cheap method to obtain a STC fluidic system on parylene $\mathrm{C}$ was presented. Based on the use of PDMS soft masks applied alternatively onto parylene C-coated silicon substrates in combination with appropriate plasma treatments, it permits to obtain a superhydrophilic pattern on a superhydrophobic background, in which pure water, water-based solutions and polar solvents can flow, confined and driven only by differences in surface energy onto the polymer substrate, without the confinement effect provided by walls that are fully missing. According to the proposed technique, fluidic systems characterized by all the desired patterns can be easily fabricated.

Most importantly, due to the strong chemical and moisture resistance of parylene $\mathrm{C}$, and to its opportunity to provide a conformal coating on a different substrate materials and shapes, the developed parylene C-based method for 2D STC fluidics is highly versatile, thus offering the opportunity to employ green, easily available and cheaper substrates, such as cellulose for paper-based fluidics applications.

\section{Acknowledgements}

We acknowledge Gianmichele Epifani from Nanotechnology Institute (CNR-NANOTEC), Lecce, Italy, for his fruitful technical help in sample preparation.

\section{Notes and references}

1 P. Tabeling, Introduction to Microfluidics, OUP, Oxford, 2010. 2 E. W. Nery and L. T. Kubota, Anal. Bioanal. Chem., 2013, 405, 7573.

3 K. Koch, B. Bhushan and W. Barthlott, Prog. Mater. Sci., 2009, 54, 137.

4 K. Koch and W. Barthlott, Philos. Trans. R. Soc., 2009, 367, 1487.

5 W. Song, D. D. Veiga, C. A. Custódio and J. F. Mano, Adv. Mater., 2009, 21, 1830.

6 S. Wang, K. Liu, X. Yao and L. Jiang, Chem. Rev., 2015, 115, 8230.

7 Y. Y. Yan, N. Gao and W. Barthlott, Adv. Colloid Interface Sci., 2011, 169, 80. 
8 M. J. Hancock, K. Sekeroglu and M. C. Demirel, Adv. Funct. Mater., 2012, 22, 2223.

9 X. Yao, Y. Song and L. Jiang, Adv. Mater., 2011, 23, 719.

10 X. Feng and L. Jiang, Adv. Mater., 2006, 18, 3063.

11 D. Quéré, Rep. Prog. Phys., 2005, 68, 2495.

12 E. Ueda and P. A. Levkin, Adv. Mater., 2013, 25, 1234.

13 J. Drelich, E. Chibowski, D. D. Meng and K. Terpilowski, Soft Matter, 2011, 7, 9804.

14 E. Ueda and P. A. Levkin, Adv. Healthcare Mater., 2013, 2, 1425.

15 Y. Han, P. A. Levkin, I. Abarientos, H. Liu, F. Svec and J. M. Fréchet, J. Anal. Chem., 2010, 82, 2520.

16 J. S. Li, E. Ueda, A. Nallapaneni, L. X. Li and P. A. Levkin, Langmuir, 2012, 28, 8286.

17 I. You, N. Yun and H. Lee, ChemPhysChem, 2013, 14, 471.

18 D. Zahner, J. Abagat, F. Svec, J. M. J. Fréchet and P. A. Levkin, Adv. Mater., 2011, 23, 3030.

19 J.-T. Lee, M. C. George, J. S. Moore and P. V. Braun, J. Am. Chem. Soc., 2009, 131, 11294.

20 M. Kaltenbrunner, T. Sekitani, J. Reeder, T. Yokota, K. Kuribara, T. Tokuhara, M. Drack, R. Schwodiauer, I. Graz, S. Bauer-Gogonea, S. Bauer and T. Someya, Nature, 2013, 499, 458.

21 N. Stark, Medical Plastics and Biomaterials Magazine, 1996, vol. 3, p. 30 .

22 Y. Chen, W. Pei, R. Tang, S. Chen and H. Chen, Sens. Actuators, A, 2013, 189, 143.

23 T. Prodromakis, K. Michelakis, T. Zoumpoulidis, R. Dekker and C. Toumazou, Proc. IEEE Sens. 2003, IEEE Int. Conf. Sens., 2nd, 2009, 746.

24 F. Rizzi, A. Qualtieri, L. D. Chambers, W. M. Megill and M. De Vittorio, Soft Matter, 2013, 9, 2584.

25 P. Calcagnile, L. Blasi, F. Rizzi, A. Qualtieri, A. Athanassiou, E. Gogolides and M. De Vittorio, ACS Appl. Mater. Interfaces, 2014, 6, 15708.

26 A. Ulman, An Introduction to Ultrathin Organic Films: From Langmuir-Blodgett to Self-Assembly, Academic Press, Inc, Boston, 1991.

27 I. Woodward, W. C. E. Schofield, V. Roucoules and J. P. S. Badyal, Langmuir, 2003, 19, 3432.

28 J. Wu, J. Xia, Y. Zhang, W. Lei and B. Wang, Physica E, 2010, 42, 1325.

29 J. Feng, M. T. Tuominen and J. P. Rothstein, Adv. Funct. Mater., 2011, XX, 1.

30 J. M. Ye, Q. M. Yin and Y. L. Zhou, Thin Solid Films, 2009, 517, 6012.

31 S. Nishimoto, A. Kubo, K. Nohara, X. Zhang, N. Taneichi, T. Okui, Z. Liu, Z. Nakata, H. Sakai, T. Murakami, M. Abe, T. Komine and A. Fujishima, Appl. Surf. Sci., 2009, 255, 622.

32 J. Yu, X. Zhao, Q. Zhao and G. Wang, Mater. Chem. Phys., 2001, 68, 253.

33 L. Sirghi and Y. Hatanaka, Surf. Sci., 2003, 53, 323.

34 A. Nakajima, K. Hashimoto and T. Watanabe, Langmuir, 2000, 16, 7044 .
35 P. Calcagnile, D. Fragouli, I. S. Bayer, G. C. Anyfantis, P. D. Cozzoli, R. Cingolani and A. Athanassiou, ACS Nano, 2012, 6, 5413.

36 H. Bai, L. Wang, J. Ju, R. Sun, Y. Zheng and L. Jiang, Adv. Mater., 2014, 26, 5025.

37 X. Tan, Y. Zhu, T. Shi, Z. Tang and G. Liao, J. Micromech. Microeng., 2016, 26, 115009.

38 J. Songok, M. Tuominen, H. Teisala, J. Haapanen, J. Mäkelä, J. Kuusipalo and M. Toivakka, ACS Appl. Mater. Interfaces, 2014, 6, 20060.

39 R. D'Agostino, P. Favia, C. Oehr and M. R. Wertheimer, LowTemperature Plasma Processing of Materials: Past, Present, and Future, Plasma Processes Polym., 2005, 1, 7.

40 G. Greene, G. Yao and R. Tannenbaum, Langmuir, 2003, 19, 5869.

41 E. Gogolides, V. Constantoudis, G. Kokkoris, D. Kontziampasis, K. Tsougeni, G. Boulousis, M. Vlachopoulou and A. Tserepi, J. Phys. D: Appl. Phys., 2011, 44, 174021.

42 K. Tsougeni, N. Vourdas, A. Tserepi and E. Gogolides, Langmuir, 2009, 25, 11748.

43 H. Teisala, M. Tuominen, M. Aromaa, M. Stepien, J. M. Mäkelä, J. J. Saarinen, M. Toivakka and J. Kuusipalo, Langmuir, 2012, 28, 3138.

44 L. Feng, Y. Zhang, J. Xi, Y. Zhu, N. Wang, F. Xia and L. Jiang, Langmuir, 2008, 24, 4114.

45 M. Gołda, M. Brzychczy-Włoch, M. Faryna, K. Engvall and A. Kotarba, Mater. Sci. Eng., 2013, 33, 4221.

46 J. S. Song, S. Lee, S. H. Jung, G. C. Cha and M. S. Mun, J. Appl. Polym. Sci., 2009, 112, 3677.

47 J. W. Seong, K. W. Kim, Y. W. Beag, S. K. Koh, K. H. Yoon and J. H. Lee, Thin Solid Films, 2005, 476, 386.

48 T. Hoshino, I. Saito, R. Kometani, K. Samejima, S. Matsui, T. Suzuki, K. Mabuchi and Y. X. Kato, J. Biosci. Bioeng., 2011, 113, 395.

49 T. Trantidou, T. Prodromakis and C. Toumazou, Appl. Surf. Sci., 2012, 261, 43.

50 M. J. Owen, T. M. Gentle, T. Orbeck and D. E. William, Polymer Surface Dynamics, Plenum Press, New York, 1988.

51 U. W. Gedde, Polymer, 1998, 39, 1991.

52 N. Vourdas, A. Tserepi, A. G. Boudouvis and E. Gogolides, Microelectron. Eng., 2008, 85, 1124.

53 L. Feng, Z. Zhang, Z. Mai, Y. Ma, B. Liu, L. Jiang and D. Zhu, Angew. Chem., Int. Ed., 2004, 43, 2012.

54 N. A. Patankar, Langmuir, 2004, 20, 8209.

55 G. McHale, N. J. Shirtcliffe and M. I. Newton, Analyst, 2004, 129, 284.

56 M. A. Nilsson, R. J. Daniello and J. P. Rothstein, J. Phys. D: Appl. Phys., 2010, 43, 1.

57 T. M. Schutzius, M. Elsharkawy, M. K. Tiwarib and C. M. Megaridis, Lab Chip, 2012, 12, 5237.

58 I. S. Khattab, F. Bandarkar, M. A. Abolghassemi Fakhree and A. Jouyban, Korean J. Chem. Eng., 2012, 29, 812. 